\title{
Experimental Demonstration of Catching and Releasing Functions of Artificial Cilia Using Induced Charge Electro-Osmosis
}

\author{
Hideyuki Sugioka, ${ }^{*}$ Yuki Mizuno, and Yusei Nambo \\ Department of Mechanical Systems Engineering, \\ Shinshu University 4-17-1 Wakasato, Nagano 380-8553, Japan
}

(Dated: March 31, 2020)

\begin{abstract}
Manipulation of an object is essential in microfluidic applications. In this study, we experimentally demonstrate that the artificial cilia having a face-to-face structure shows catching and releasing functions in water through induced charge electro-osmosis. In particular, by the visualization of the flow fields, we show that a target object was pulled into a cilium region by the pull-in flow due to the up-wing motion of the cilia and released by the pop-up flow due to the downwing motion. We believe that our device is useful to manipulate various particles in microfluidic channels.
\end{abstract}

PACS numbers: 47.57.-s, 85.90.+h, 82.45.Hk, 83.50.Lh

\footnotetext{
* hsugioka@shinshu-u.ac.jp
} 


\section{INTRODUCTION}

Manipulation of an object plays an important role in microfluidic applications, such as a micro total analysis systems and Lab-on-a-chip [1]. Thus, various methods have been proposed so far [2, 3]. For example, Chronis and Lee reported a SU-8-based mechanical microgripper consisting of two electrothermal actuators and demonstrated the catching function in aqueous solution [4]. Lee et al. reported manipulation of biological cells using a microelectromagnet matrix [5]. Han and Frazier demonstrated a continuous magnetophoretic separation of red and white blood cells from whole blood based on their magnetic properties [6]. Grilli and Ferraro demonstrated dielectric trapping of suspended particles by selective pyroelectric effect [7]. Duhr and Braun showed that the combination of thermophoresis and fluid flow results in strong trapping of small biomolecules [8]. Nilsson et al. showed that suspended particles can be separated from their medium by an ultrasonic standing wave field [9]. Huang et al. demonstrated continuous particle separation through deterministic lateral displacement due to the array of posts [10]. However, since above technologies usually have the only function, we need to combine different technologies to built a system and it often causes difficulties. Thus, a multi-functional fluidic device having a manipulation function with other functions, such as pumps, valves, mixers, etc., is ideally expected as a future device since it enables us to build practical integrated systems in a similar manner.

In this context, artificial cilia that mimic natural cilia are considered to be promising for constituting microfluidic systems since they are expected to have various functions [11-14]. Thus, various artificial cilia have been reported so far. For example, Vilfan et al. reported self-assembled artificial cilia using superparamagnetic colloidal particles [15]. Hanasoge et al. demonstrated microfluidic pumping of $\sim 1.35 \mathrm{~mm} / \mathrm{s}$ using artificial magnetic cilia [16]. Toonder et al. reported that electrostatic cilia having a platelike but curled microbeam consisting of layers of polyimid and chromium can be driven by an electrostatic force and generate substantial fluid flow in silicon oil [12]. Orbay et al. reported acoustic actuation of cilia structures using piezo transducers [17]. Oosten et al. reported printed artificial cilia using liquid-crystal actuators driven by light [18]. Masuda et al. reported gradient artificial cilia using a self-oscillating polymer brush [19]. Sareh et al. reported artificial cilia using a multi-segment ionic polymer metal composite (IPMC) actuator and demonstrated ciliumlike motions [20]. However, in spite of its potentiality, each technology seems to have subjects 
to overcome. For example, the whole miniaturization of magnetic cilia seems to be difficult since they require a large magnetic device, whereas Toonder et al.'s electrostatic cilia work only in oil and thus it limits their applications. Furthermore, it might be difficult to control specific area without affecting the other area for acoustic cilia, whereas self-oscillating cilia and light-driven cilia need to find a suitable operation method for their practical use. In addition, although IPMC cilia are promising for miniaturization and operability in water, they seem to require tremendous connecting wires for huge numbers of cilia. Therefore, we need to continue to explore new class of cilia. In particular, we consider that ideal cilia should have following characteristics: (1) possibility of total miniaturization, (2) operability in water, and (3) low number of connections.

To obtain the ideal cilia, we have theoretically proposed a valve [21], a pump [22], and a catcher [23] using the ICEO cilia on the basis of the multi-physics calculation method, and experimentally demonstrated the basic beating motions [24, 25]. Here, artificial cilia using induced charge electro-osmosis (ICEO) are challenging since they can be driven in water by small voltage $(\sim \mathrm{V})[21-23]$ and thus we can realize a miniaturized integrated system that works with a battery with the proper number of connections in the future. However, we have not reported the catching and releasing motions of the ICEO cilia experimentally, yet. Therefore, in this study, we experimentally demonstrate that the artificial cilia having a face-to-face structure show catching and releasing functions in water through induced charge electro-osmosis. In particular, by the visualization of the flow fields, we show that a target object was pulled into a cilium region by the pull-in flow due to the up-wing motion of the cilia and released by the pop-up flow due to the down-wing motion, as predicted in our previous paper [23].

\section{METHOD}

Figure 1 shows an experimental setup of catching and releasing functions of the ICEO cilia having a face-to-face structure. As shown in Fig. 1(a), we set a pair of beams of width $w_{2}(=7 \mathrm{~mm})$, length $l(=15 \mathrm{~mm})$, and thickness $d_{b}(=0.01 \mathrm{~mm})$ on the lower Cu electrode of length $L_{1}(=47 \mathrm{~mm})$ and placed the upper $\mathrm{Cu}$ electrode of length $L_{2}(=30 \mathrm{~mm})$. In Fig. 1(a), $\boldsymbol{x}_{b R}\left[=\left(x_{b R}, y_{b R}\right)\right]$ and $\boldsymbol{x}_{b L}\left[=\left(x_{b L}, y_{b L}\right)\right]$ denote the left and right edge positions,

respectively, whereas $\boldsymbol{x}_{p}\left[=\left(x_{p}, y_{p}\right)\right]$ denotes the edge of a target object (square polyethylene 

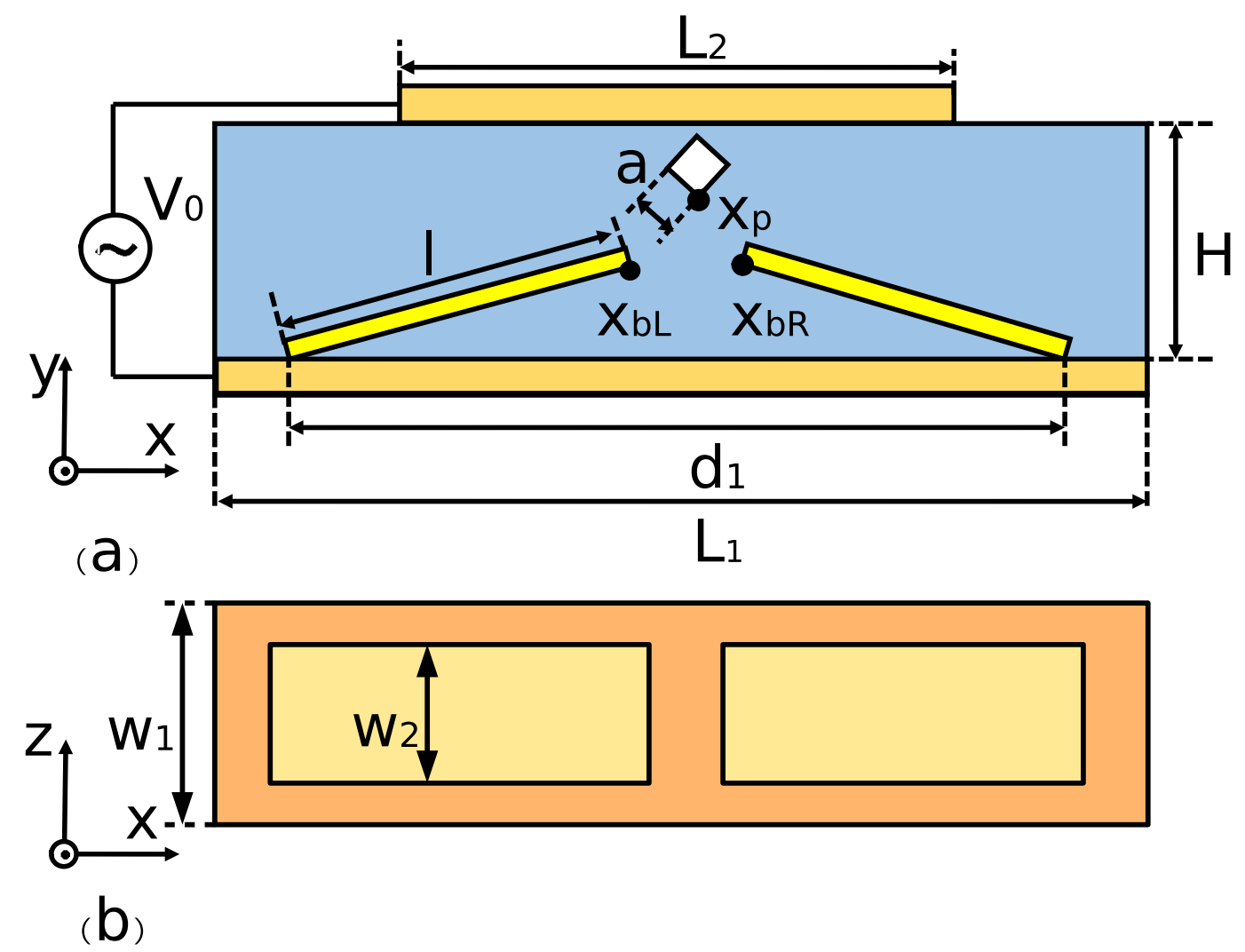

FIG. 1. (Color online) Experimental setup of catching and releasing functions of the ICEO cilia having a face-to-face structure. (a) Side view. (b) Top view. Here, $a=1.5 \mathrm{~mm}, l=15 \mathrm{~mm}, H=4$ $\mathrm{mm}, L_{1}=47 \mathrm{~mm}, L_{2}=30 \mathrm{~mm}, d_{1}=32 \mathrm{~mm}, d_{2}(0)=x_{b R}(0)-x_{b L}(0) \simeq 1.36 \mathrm{~mm}, w_{1}=12 \mathrm{~mm}$, and $w_{2}=7 \mathrm{~mm}$.

film) of size $a \times a$ and thickness $d_{p}$, where $a=1.5 \mathrm{~mm}$ and $d_{p}=0.01 \mathrm{~mm}$. Further, as shown in Fig. 1(a), $w_{1}(=12 \mathrm{~mm})$ denotes the width of the chamber and $w_{2}(=7 \mathrm{~mm})$ denotes the width of the beams. Here, the beams were made of polyethylene film and it was coated by an Au film of thickness $100 \mathrm{~nm}$ to obtain a large deformation [26]. After injecting water (milli-Q, $18.2 \mathrm{M} \Omega \mathrm{cm}$ initially) into the chamber of height $H(=4 \mathrm{~mm})$, we applied a rectangular $\mathrm{AC}$ voltage of peak value $V_{0}$ and frequency $f$ between the upper and lower electrodes at $t=0$ and turned off the $\mathrm{AC}$ voltage at $t=t_{\text {off }}$, where $t$ is a time. Then, we observed the motions of the beams and the object. Here, we repeated the observation $N_{f}$ times (typically, $N_{f}=3$ ) under the same conditions. By using the recorded video data of size $640 \times 480$ (or $1920 \times 1080)$ with a frame rate of 30 (or 60$)$ fps, we determined the edge positions $\left[\boldsymbol{x}_{b R}(t), \boldsymbol{x}_{b L}(t)\right.$, and $\left.\boldsymbol{x}_{p}(t)\right]$. Furthermore, to visualize flow fields, we used 
pearl pigment as a tracer and determined the flow fields $\boldsymbol{u}=\left(u_{x}(x, y, t), u_{y}(x, y, t)\right)$ by the particle image velocimetry. Further, we define $U_{y}$ as the minimum value of $u_{y}$ at $t<t_{\text {off }}$ in the region $D$ to evaluate a pull-in flow during the catching motion, whereas we define $U_{y}$ as the minimum value of $u_{y}$ at $t<t_{o f f}$ in the region $D$ to evaluate a pop-up flow during the releasing motion, where $D=\{(x, y) \mid-0.68 \leq x \leq+0.68 \mathrm{~mm}, 0 \leq y \leq 4 \mathrm{~mm}\}$, and the origin of the coordinate is the mid point on the surface of the electrode. Further, to evaluate the pull-in and pop-up values for the object, we define that $\Delta y_{p, \max 1}=y_{p}(0)-y_{p}\left(t_{o f f}\right)$ and $\Delta y_{p, \max 2}=y_{p}\left(t_{f}\right)-y_{p}\left(t_{o f f}\right)$, whereas we define that $\Delta y_{b, \max 1}=y_{b}\left(t_{o f f}\right)-y_{b}(0)$ and $\Delta y_{b, \max 2}=y_{p}\left(t_{o f f}\right)-y_{p}\left(t_{f}\right)$, to evaluate the pull-in and pop-up values for the beam, where $t_{f}$ is the final time of the measurement.

\section{RESULTS}

Figures 2(a), 2(b), 2(c), 2(d), 2(e), and 2(f) show the photographs of the typical catching and releasing motions at $t=0.067,0.40,0.70,1.67,1.73$, and $2.03 \mathrm{~s}$ when we used pearl pigment for visualization. Here, $t_{o f f}=1.5 \mathrm{~s}, V_{0}=40 \mathrm{~V}, f=100 \mathrm{~Hz}, y_{b L}(0)=2.00 \mathrm{~mm}$, $y_{b R}(0)=1.94 \mathrm{~mm}$, and $y_{p}(0)=3.47 \mathrm{~mm}$. By applying an AC electric field, we observed that the left and right beams start to move in the upper direction by the ICEO down-flows at the edges at $t=0.067 \mathrm{~s}$ [Fig. 2(a)], as predicted in our previous paper [23]. As a result, the beams moved toward the upper electrode owing to the ICEO down flows and thus the target object experienced a large pull-in flow $\left(U_{y}=-2.6 \mathrm{~mm} / \mathrm{s}\right)$ at $t=0.40 \mathrm{~s}$ [Fig. 2(b)]. Consequently, the object was caught in a cilium region at $t=0.70 \mathrm{~s}$ [Fig. 2(c)]. By turning off the AC electric field at $t=t_{o f f}(=1.5 \mathrm{~s})$, we observed that the left and right beams started to the lower direction by the elastic forces of the beams at $t=1.67 \mathrm{~s}$ [Fig. 2(d)]. As a result, the object experienced a large pop-up flow $\left(U_{y}=+4.2 \mathrm{~mm} / \mathrm{s}\right)$ at $t=1.73 \mathrm{~s}$ [Fig. 2(e)]. Consequently, the object was released at $t=2.03 \mathrm{~s}$ [Fig. 2(f)].

Figure 3 shows the characteristics of the catching and releasing motions when we used pearl pigment for visualization. Specifically, Fig. 3(a) shows the dependence of $y_{b}$ on $t$. As shown in Fig. 3(a), the left and right beams reached the upper electrode at $t \simeq 0.8$ and $1.2 \mathrm{~s}$, respectively, whereas the beams returned to the initial positions during $\sim 0.5 \mathrm{~s}$. Here, the difference of the response times was due to the difference of $y_{b}(0)$ [i.e., $y_{b L}(0)=2.00$ $\mathrm{mm}$ and $\left.y_{b R}(0)=1.94 \mathrm{~mm}\right]$. Figure $3(\mathrm{~b})$ shows the dependence of $y_{p}$ on $t$. From Fig. 3(a), 


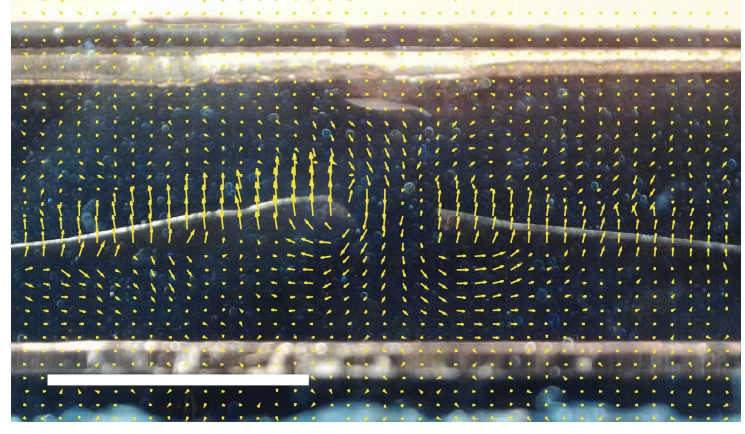

(a) $t=0.067 \mathrm{~s}$

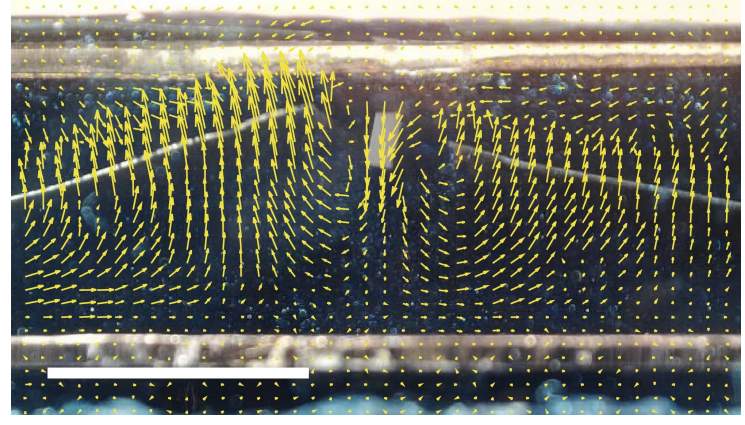

(c) $t=0.70 \mathrm{~s}$

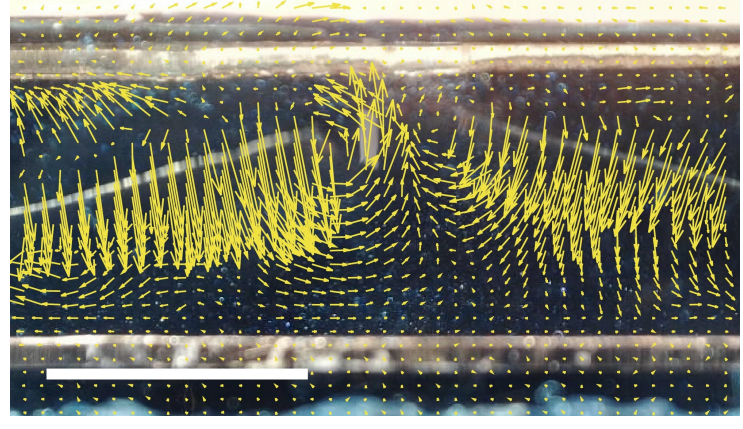

(e) $t=1.73 \mathrm{~s}$

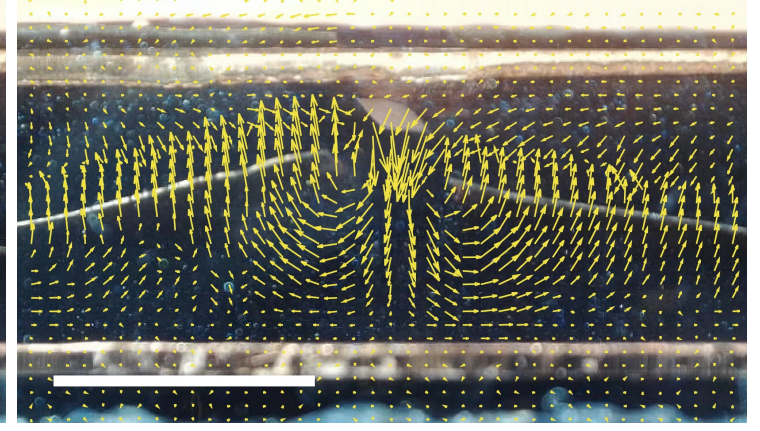

(b) $t=0.40 \mathrm{~s}$

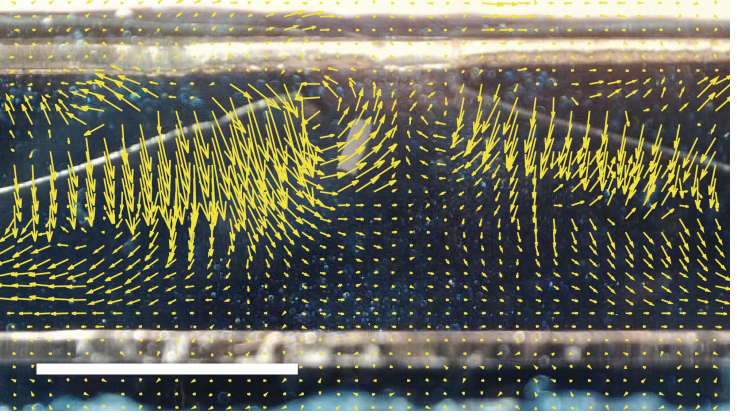

(d) $t=1.67 \mathrm{~s}$

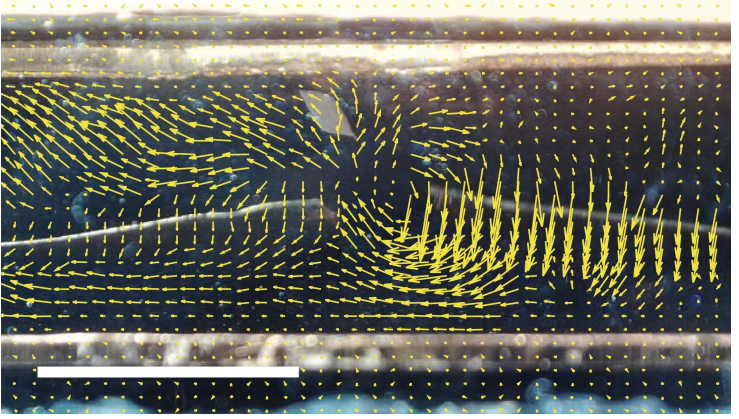

(f) $t=2.03 \mathrm{~s}$

FIG. 2. (Color online) Catching and releasing flows due to artificial cilia using ICEO with pearl pigment. Here, we use the video data of size $640 \times 480$ with a frame rate of $30 \mathrm{fps} ; V_{0}=40 \mathrm{~V}$, $f=100 \mathrm{~Hz}, y_{b L}(0)=2.00 \mathrm{~mm}, y_{b R}(0)=1.94 \mathrm{~mm}$, and $y_{p}(0)=3.47 \mathrm{~mm}$; the scale bar shows 4 $\mathrm{mm}$.

we found that the target object was pulled into the lower direction by the up-wing motions of the beams, whereas the object was popped up by the down-wing motions. Figure 3(c) shows the dependence of $U_{y}$ on $t$. From Figs. 3(c), we found that a strong pull-in flow $\left(U_{y} \simeq-1\right.$ to $\left.-4 \mathrm{~mm} / \mathrm{s}\right)$ was generated by the up-wing motions at $t \sim 0.5 \mathrm{~s}$, whereas a strong pop-up flow $\left(U_{y} \simeq+3\right.$ to $\left.+4 \mathrm{~mm} / \mathrm{s}\right)$ was generated by the down-wing motions at $t \sim 1.7 \mathrm{~s}$. 


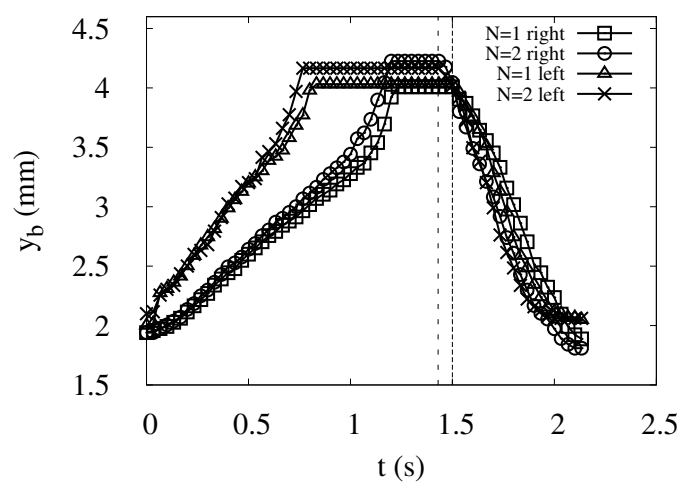

(a) Dependence of $y_{b}$ on $t\left(V_{0}=40 \mathrm{~V}\right)$

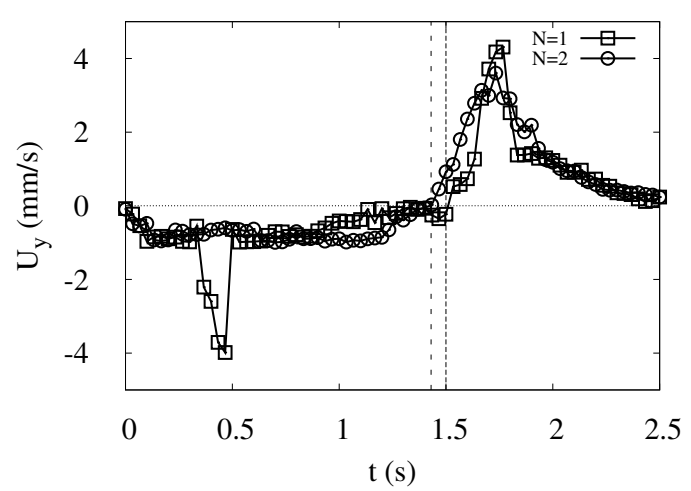

(c) Dependence of $U_{y}$ on $t\left(V_{0}=40 \mathrm{~V}\right)$

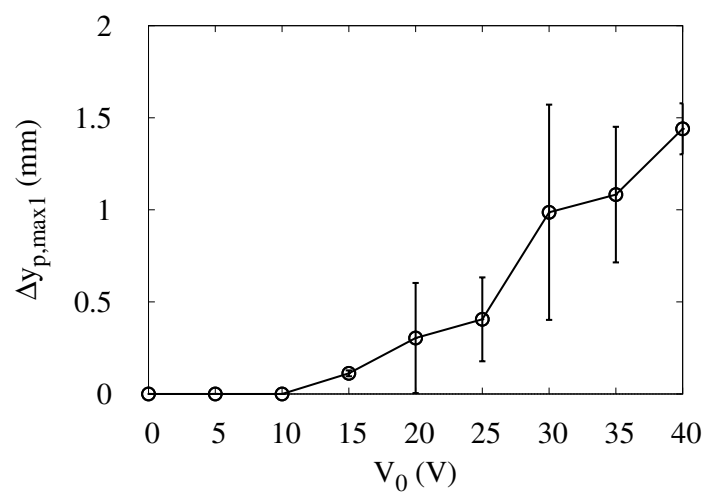

(e) Dependence of $\Delta y_{p, \max 1}$ on $V_{0}(f=100 \mathrm{~Hz})$

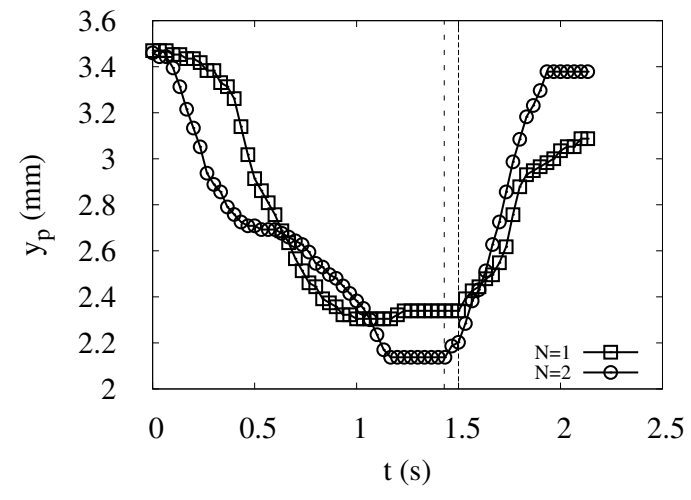

(b) Dependence of $y_{p}$ on $t\left(V_{0}=40 \mathrm{~V}\right)$

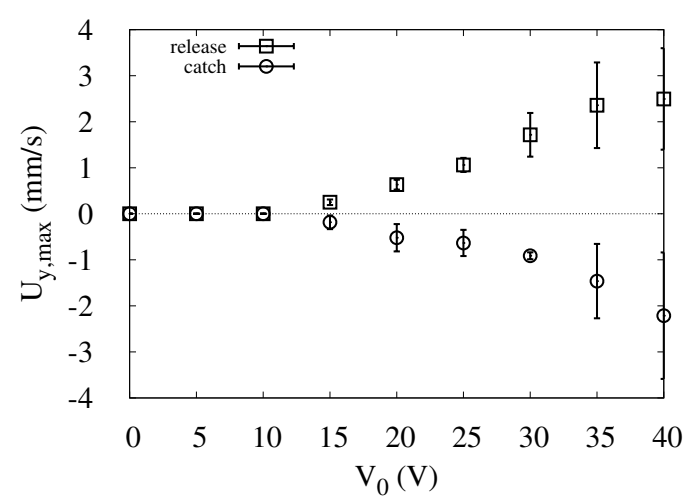

(d) Dependence of $U_{y, \max }$ on $V_{0}(f=100 \mathrm{~Hz})$

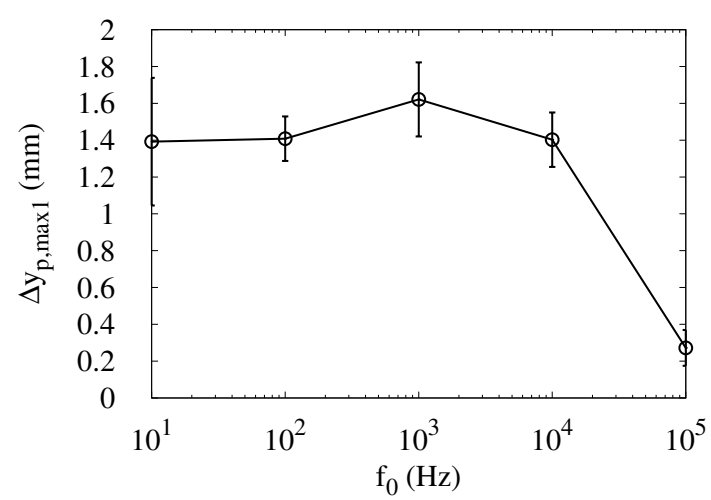

(f) Dependence of $\Delta y_{p, \max 2}$ on $f\left(V_{0}=40 \mathrm{~V}\right)$

FIG. 3. Characteristics of the catching and releasing motions when we used pearl pigment for visualization. In (a) to (c), $V_{0}=40 \mathrm{~V}$ and $f=100 \mathrm{~Hz}$. In (a), $t_{\text {off }}=1$ and $1.5 \mathrm{~s}$ for $N=1$ and 2. Here, we use the video data of size $640 \times 480$ with a frame rate of $30 \mathrm{fps}$. 

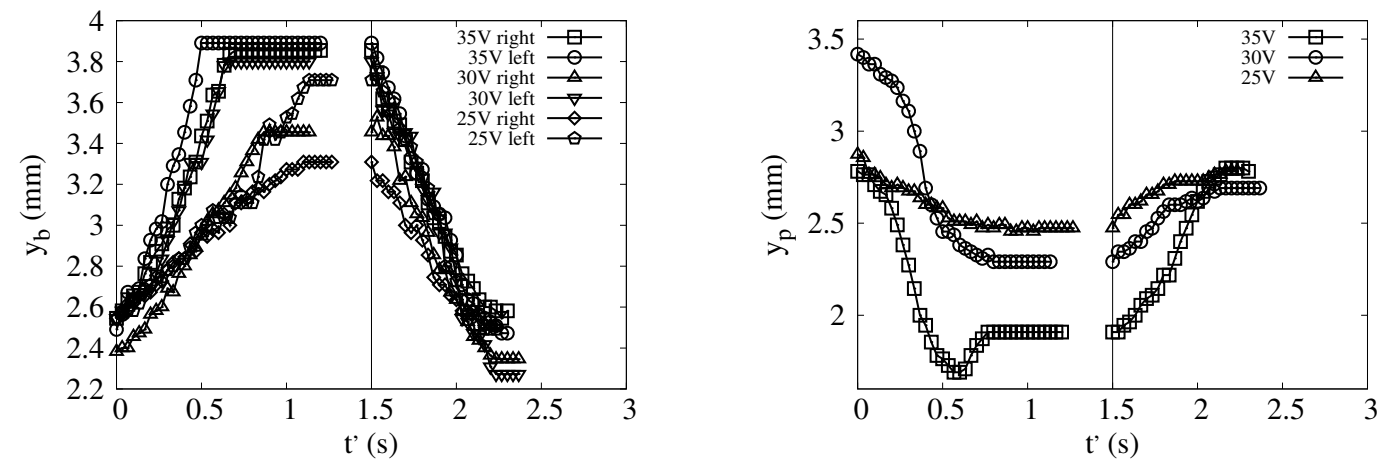

(a) Dependence of $y_{b}$ on $t^{\prime}(f=100 \mathrm{~Hz}, N=1)$

(b) Dependence of $y_{p}$ on $t^{\prime}(f=100 \mathrm{~Hz}, N=1)$
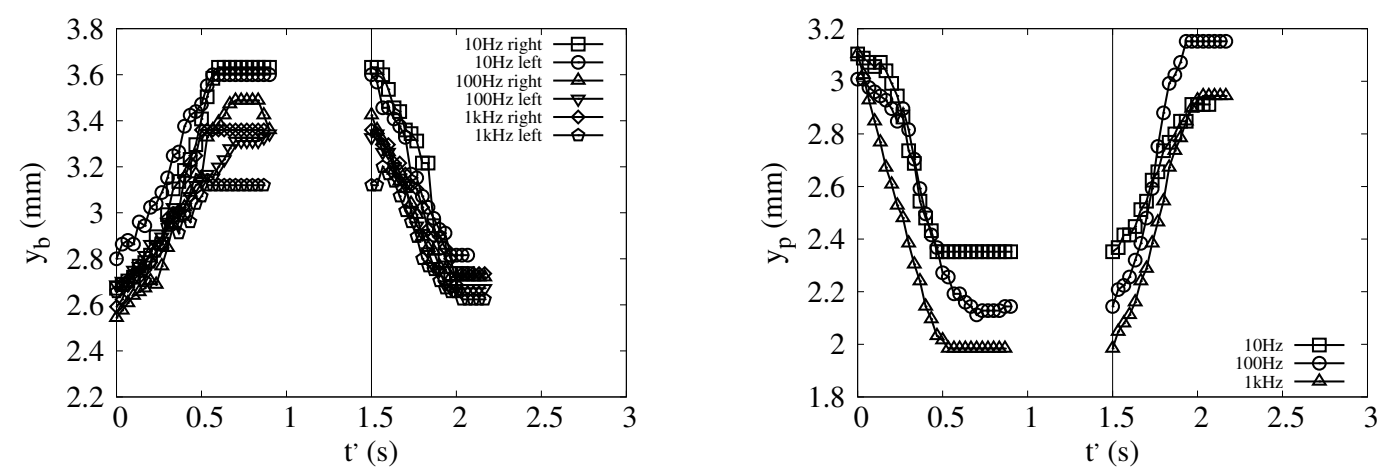

(c) Dependence of $y_{b}$ on $t^{\prime}\left(V_{0}=35 \mathrm{~V}, N=1\right)$

(d) Dependence of $y_{p}$ on $t^{\prime}\left(V_{0}=35 \mathrm{~V}, N=1\right)$
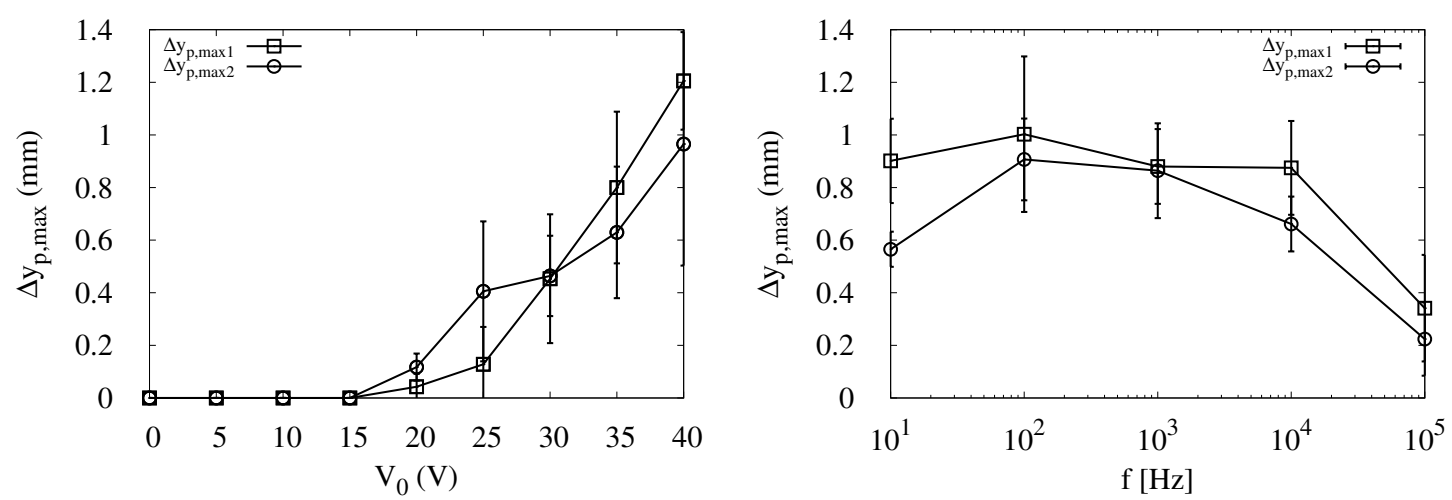

(e) Dependence of $\Delta y_{p, \max }$ on $V_{0}(f=100 \mathrm{~Hz})$

(f) Dependence of $\Delta y_{p, \max }$ on $f\left(V_{0}=35 \mathrm{~V}\right)$

FIG. 4. Characteristics of the catching and releasing motions without pearl pigment. Here, we used the video data of size $1920 \times 1080$ with a frame rate of $60 \mathrm{fps}$; in (e) and (f), $N_{f}=3$.

Figure 3(d) shows the dependence of $U_{y, \max }$ on $V_{0}$ during the catching and releasing motions, where $U_{y, \max }$ denotes the minimum (maximum) value at $t<t_{\text {off }}\left(t>t_{\text {off }}\right)$. In Fig. $3(\mathrm{~d})$, we found the nonlinearity corresponding to the ICEO characteristic. Figures 3(e) and 3(f) 
show the dependences of $\Delta y_{p, \max 1}$ on $V_{0}$ and $f$, respectively. In Fig. 3(e), we also found the nonlinearity corresponding to the ICEO characteristic. Further, from Fig. 3(f), we found that $\Delta y_{p, \max 1}$ was approximately $0.6 \mathrm{~mm}$ at $10^{1} \leq f \leq 10^{4} \mathrm{~Hz}$ and decreased at $f>10^{4} \mathrm{~Hz}$ because of the insufficient charging time.

Figure 4 shows the characteristics of the catching and releasing motions without pearl pigment. Figures 4(a) and 4(b) [Figures 4(c) and 4(d)] show the dependences of $y_{b}$ and $y_{p}$ on $t^{\prime}$ under the condition that $V_{0}=25$ to $35 \mathrm{~V}$ and $f=100 \mathrm{~Hz}\left[V_{0}=35 \mathrm{~V}\right.$ and $f=10$ to $1000 \mathrm{~Hz}]$. Here, we defined $t^{\prime}$ as $t^{\prime}=t$ at $t \leq t_{\text {off }}$ and $t^{\prime}=t-t_{\text {off }}+1.5$ at $t \geq t_{\text {off }}$. Figure $4(\mathrm{e})$ shows the dependences of $\Delta y_{b, \max 1}$ and $\Delta y_{b, \max 2}$ on $V_{0}$, whereas Fig. $4(\mathrm{f})$ shows the dependences of $\Delta y_{p, \max 1}$ and $\Delta y_{p, \max 2}$ on $f$. From these Figures, we recognized that the catching and releasing motions were usually observed under the condition that $V_{0}=25$ to $40 \mathrm{~V}$ and $f=10$ to $10000 \mathrm{~Hz}$. Furthermore, from Figs. 3 and 4, we found that there was a tendency that the pull-in values with pearl pigment were slightly larger than the pull-in values without pearl pigment under the same condition. As a result, the object was often pulled into the side part of the cilium region deeply and it cannot be popped up when we used pearl pigment. This is probably because the beams move well in the $y$ direction when there is pearl pigment, because the increasing of the specific gravity due to the pigment results in the increasing of the buoyancy of the beams.

\section{DISCUSSION}

\section{A. Meaning of this study}

In this study, we have experimentally demonstrated that the artificial cilia having a faceto-face structure shows catching and releasing functions in water through induced charge electro-osmosis, although we theoretically predicted the catching and releasing function in our previous paper [23]. In particular, by the visualization of the flow fields, we show that a target object was pulled into a cilium region by the pull-in flow due to the up-wing motion of the cilia and released by the pop-up flow due to the down-wing motion. Although mechanical tweezers are generally considered to fail in manipulating particles in a low Reynolds regime [4], the motions of our ICEO cilia can operate flows by the motion of their beams and thus they can manipulate the object near the beams. In detail, as pointed out by Chronis and 
Lee [4], the motion of the microgripper creates fluid motion that drags the object away. Therefore, our device is useful to manipulate various objects in microfluidic channels. In addition, since ICEO cilia potentially have the characteristics of the ideal cilia mentioned in Sec. I, they are promising as the future microfluidic device.

\section{B. About the large fluctuation in the dependence of $U_{y}$ on $t$}

Although the behaviors of $y_{b}$ at $N=1$ and 2 in Fig. 3(a) are similar, the behaviors of $y_{p}$ and $U_{y}$ in Figs. 3(b) and 3(c) are definitely different between $N=1$ and 2. This is because the target sheet is initially in a horizontal state and change the attitude with large fluctuation to be in a vertical state in a flow to reduce flow resistance. In detail, the target sheet at $N=2$ starts to change the attitude at $t \simeq 0 \mathrm{~s}$ and ends the change at at $t \simeq 0.4$ s, whereas the target sheet at $N=1$ starts to change the attitude at $t \simeq 0.4$, in Fig. $3(\mathrm{~b})$. By this difference, the values of $U_{y}$ at $N=1$ and 2 become approximately 4 and $1 \mathrm{~mm} / \mathrm{s}$, respectively, at $t \simeq 0.4 \mathrm{~s}$. In other words, because of the rotation of the objects, the value of $U_{y}$ sometimes fluctuates. However, since the behavior of $U_{y}$ corresponds to that of $y_{p}$, our measurements are reasonable.

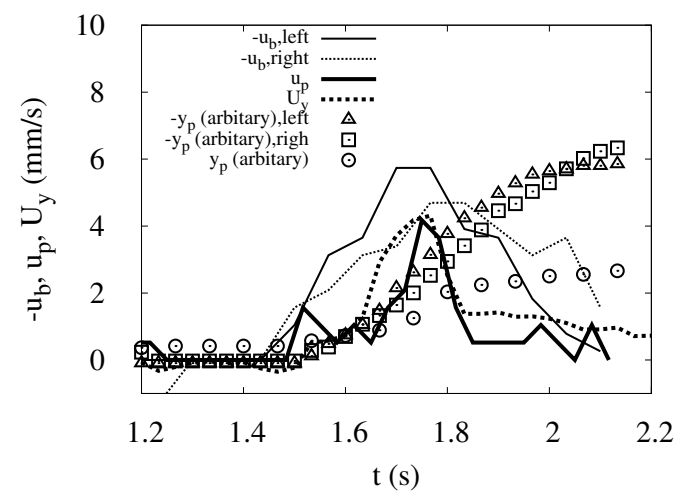

(a) $N=1$

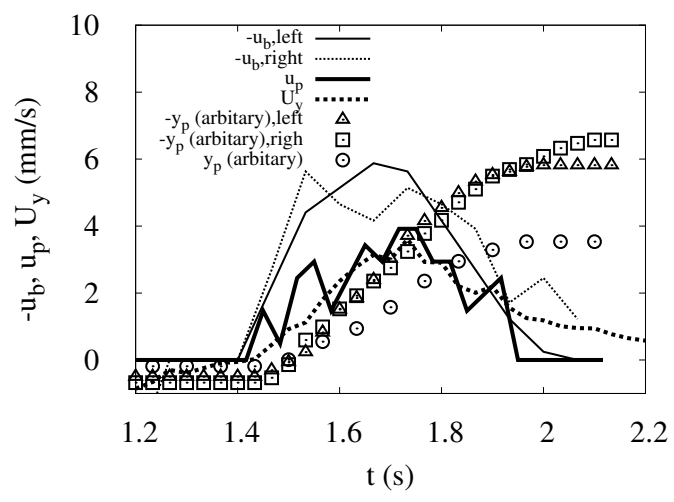

(b) $N=2$

FIG. 5. Dependences of $-u_{b}, u_{p}$, and $U_{y}$ on $t$. Here, $V_{0}=40 \mathrm{~V}$ and $f=100 \mathrm{~Hz}$; we used the video data of size $640 \times 480$ with a frame rate of $30 \mathrm{fps}$. 


\section{About the relation between $u_{b}\left(\equiv \frac{d y_{b}}{d t}\right)$ and $U_{y}$ during the down-wing motion}

In a rough evaluation, the data of Fig. 3(a) show that the velocity of the object is approximately $4 \mathrm{~mm} / \mathrm{s}$ during down-wing motion $(1.5 \leq t \leq 2 \mathrm{~s})$, whereas the maximum flow velocity is $4 \mathrm{~mm} / \mathrm{s}$ only for the short time at $t \simeq 1.7 \mathrm{~s}$. Although it seems to be strange, it is reasonable because the detail analysis in Fig. 5 shows that the velocity of the beams $u_{b}\left(\equiv \frac{d y_{b}}{d t}\right)$ is initially small and gradually becomes large with $t$. Then, $u_{b}$ gradually becomes small again during down-wing motion. That is, from Fig. 5, we find that $u_{b}$ has a strong correlation with $u_{p}$ and $U_{y}$. Therefore, the fundamental behavior of $U_{y}$ during down-wing motion in Fig. 3(c) is reasonable. Physically speaking, the down-wing motion occurs by the elastic force $f_{e}$ in the presence of viscous force $f_{v}$. Thus, $u_{b}$ is small in the initial stage and gradually becomes large until $f_{e}$ is in balance with $f_{v}$. Then, $u_{b}$ becomes small again, because $f_{e}$ becomes small as $\theta$ becomes small. However, the period having large $U_{y}$ seems to be smaller significantly than the period having large $u_{b}$ at $N=1$, in Fig. 5(a). This is because $u_{b}$ does not directly affect $U_{y}$. In other words, the period having large $u_{p}$ and $U_{y}$ must be the region that both left and right $u_{b}$ values are large since they affect $u_{p}$ and $U_{y}$. Thus, the overlapped region that the left and right $u_{b}$ values are large has large $u_{p}$ and $U_{y}$ values in Fig. 5(a).

\section{Future work}

It might be important to measure the change of the electric current accompanied by the application of the $\mathrm{AC}$ voltage, since it would provide us important information on the underlying mechanism of the motion of artificial cilia. However, there remain many fundamental issues unclear for the dielectric spectroscopy of ICEO cilia, although this kind technique are investigated extensively as dielectric spectroscopy or impedance spectroscopy [27]. For example, (1) dielectric spectroscopy that considers electric double layers at large applied voltages between simple parallel electrodes should be established, although many challenging studies exist [28-31]. In particular, although the RC model using the modified Poisson-Boltzmann (M-PB) theory [28] might be considered as a major theory to explain a current response of an electric double layer of the simple electrolyte-electrode system, we consider that the dielectric spectroscopy using the ion-conserving (IC) M-PB or IC-PB 
theory $[32,33]$ should be developed for a practical analysis, since the M-PB theory neglects the ion-conserving condition. (2) RC models considering Faraday current should be established since there exist many practical systems involving Faraday current [29]. Note that the behavior of the Faraday current is very complex since the condition of the diffused layer in electric double layer changes dramatically by the applied voltages $[29,34,35]$. Thus, RC models considering Faraday current is challenging. (3) Low-frequency dielectric dispersion (or $\alpha$ dispersion) phenomenon [36] should be clarified in ICEO systems, since it may cause large phase delay and affect the current [37]. Note that $\alpha$ dispersion is usually known as a phenomenon that causes a significant frequency dependence in the low-frequency dielectric constant [36]. Thus, the study of $\alpha$ dispersion in ICEO systems is essential to understand the meaning of the current. (4) Current theory for the ICEO cilia should be established along with new calculation technique, although the multi-physics calculation method based on the boundary element method combined with the thin-double-layer approximation might be used to some extent. Note that the precise direct calculation based on the finite-element method (FEM) combined with finite volume method [37] is very difficult for the ICEO cilia, because it requires reconstruction of the FEM mesh structure at each time step for the thin electric-double-layer region. Because of those reasons, the dielectric spectroscopy of ICEO cilia is very challenging. Thus, we would like to clarify the current problem of ICEO cilia in the future, although it is beyond the scope of this manuscript.

\section{CONCLUSION}

In conclusion, we have experimentally demonstrated catching and releasing functions due to the ICEO cilia in water under an AC electric field. Furthermore, by the visualization of the flow fields, we show that those functions were achieved by the pull-in and pop-up flows due to the up- and down-wing motions of the ICEO cilia.

[1] T. Squires and S. Quake, Rev.Mod.Phys. 77, 977 (2005).

[2] C. Yi, C.-W. Li, S. Ji, and M. Yang, Analytica Chimica Acta 560, 1 (2006).

[3] C. Zhang, K. Khoshmanesh, A. Mitchell, and K. Kalantar-zadeh, Analytical and Bioanalytical Chemistry 396, 401 (2010). 
[4] N. Chronis and L. P. Lee, JOURNAL OF MICROELECTROMECHANICAL SYSTEMS 15, 857 (2005).

[5] H. Lee, A. M. Purdon, and R. M. Westervelt, Applied Physics Letters 85, 1063 (2004).

[6] K.-H. Han and A. Bruno Frazier, Journal of Applied Physics 96, 5797 (2004).

[7] S. Grilli and P. Ferraro, Applied Physics Letters 92, 232902 (2008).

[8] S. Duhr and D. Braun, Phys. Rev. Lett. 97, 038103 (2006).

[9] A. Nilsson, F. Petersson, H. Jnsson, and T. Laurell, Lab Chip 4, 131 (2004).

[10] L. R. Huang, E. C. Cox, R. H. Austin, and J. C. Sturm, Science 304, 987 (2004).

[11] J. den Toonder and P. Onck, Trends Biotechnol. 31, 85 (2013).

[12] J. d. Toonder, F. Bos, D. Broer, L. Filippini, M. Gillies, J. de Goede, T. Mol, M. Reijme, W. Talen, H. Wilderbeek, V. Khatavkar, and P. Anderson, Lab Chip 8, 533 (2008).

[13] S. N. Khaderi, J. M. J. den Toonder, and P. R. Onck, Journal of Fluid Mechanics 688, 4465 (2011).

[14] A. Shields, B. Fiser, B. Evans, M. Falvo, S. Washburn, and R. Superfine, PNAS 107, 15670 (2010).

[15] M. Vilfan, A. Potočnik, B. Kavčič, N. Osterman, I. Poberaj, A. Vilfan, and D. Babič, Proceedings of the National Academy of Sciences 107, 1844 (2010).

[16] S. Hanasoge, P. Hesketh, and A. Alexeev, Microsystems Nanoengineering 4, 11 (2018).

[17] S. Orbay, A. Ozcelik, H. Bachman, and T. J. Huang, Journal of Micromechanics and Microengineering 28, 025012 (2018).

[18] C. L. van Oosten, C. W. M. Bastiaansen, and D. J. Broer, Nature Materials 8, 677 (2009).

[19] T. Masuda, A. M. Akimoto, K. Nagase, T. Okano, and R. Yoshida, Science Advances 2 (2016).

[20] S. Sareh, J. Rossiter, A. Conn, K. Drescher, and R. E. Goldstein, Journal of The Royal Society Interface 10, 20120666 (2013).

[21] H. Sugioka, Phys. Rev. Applied 3, 064001 (2015).

[22] H. Sugioka, Journal of the Physical Society of Japan 85, 104001 (2016).

[23] H. Sugioka, Journal of the Physical Society of Japan 86, 014401 (2017).

[24] H. Sugioka and N. Nakano, Phys. Rev. E 97, 013105 (2018).

[25] H. Sugioka, N. Nakano, and Y. Mizuno, Journal of the Physical Society of Japan 88, 084801 (2019). 
[26] H. Sugioka and Y. Mizuno, Journal of the Physical Society of Japan 89, 044401 (2020).

[27] L. A. Rosen and D. A. Seville, Langmuir 7, 36 (1991).

[28] M. S. Kilic, M. Z. Bazant, and A. Ajdari, Phys. Rev. E 75, 021502 (2007).

[29] M. Soestbergen, P. Biesheuvel, and M. Bazant, Phys. Rev. E 81, 021503 (2010).

[30] L. H. Olesen, M. Z. Bazant, and H. Bruus, Phys. Rev. E 82, 011501 (2010).

[31] H. Sugioka, Phys. Rev. E 86, 016318 (2012).

[32] H. Sugioka, Journal of the Physical Society of Japan 85, 124006 (2016).

[33] H. Sugioka, Journal of the Physical Society of Japan 84, 104001 (2015).

[34] D. C. Prieve, Colloids and Surfaces A: Physicochemical and Engineering Aspects 250, 67 (2004).

[35] Y. Kim and D. F. Lawler, Desalination 285, 245 (2012).

[36] A. Delgado, F. Arroyo, F. Gonzlez-Caballero, V. Shilov, and Y. Borkovskaya, Colloids and Surfaces A: Physicochemical and Engineering Aspects 140, 139 (1998).

[37] H. Sugioka, Phys. Rev. E 94, 022609 (2016). 